\title{
THEORY OF THE SYNCHRONOUS MOTOR.
}

\author{
BY CHARLES PROTEUS STEINMETZ.
}

The following theory of the synchronous motor was written somewhat over a year ago, but was not intended for publication. Since, however, through Prof. S. P. Thompson's paper, entitled "Some Advantages of Alternating Currents," read before the British Association, the synchronous motor has been brought into discussion again, it may be of interest to communicate this sketch.

While by the use of the method of complex quantities, the analytical treatment may be shortened somewhat, I consider it preferable to give the theory essentially in its original form.

I shall discuss one circuit only; the results apply however to the polyphase synchronous motor as well. In the latter case the volts, amperes, watts, etc., are these quantities per phase of the system, so that for instance in a three-phase synchronous motor the total power is three times the value per phase, introduced in the discussion.

Let $u=\sqrt{r^{2}+s^{2}}=$ impedance of the circuit of (equivalent) resistance $r$ and (equivalent) reactance $s=2 \pi N L$, containing the impressed E. M. F. $e_{0}{ }^{1}$ and the counter E. M. F. $e_{1}$ of the synchronous motor, that is the E. M. F. induced in the motor armature by its rotation through the (resultant) magnetic tield.

Let $c=$ current in the circuit (effective values).

1. If $e_{0}=$ E. M. F. at motor terminals, $u=$ internal impedances of the motor; if $e_{0}=$ terminal voltage of the generator, $u=$ total impedance of line and motor; if $e_{0}=$ E. M. F of generator, that is, E. M. F. induced in generator armature by its rotation through the magnetic field, $u$ includes the generator ímpedance also. 
The mechanical power delivered by the synchronous motor (including friction and core loss), is the electric power consumed by the c. E. M. F. $e_{1}$, hence:

$$
p=c e_{1} \cos \left(c_{1} e_{1}\right)
$$

thus :

$$
\left.\begin{array}{l}
\cos \left(c_{1} e_{1}\right)=\frac{p}{c e_{1}} \\
\sin \left(c_{1} e_{1}\right)=\sqrt{1-\left(\frac{p}{c e_{1}}\right)^{2}} .
\end{array}\right\}
$$

The displacement of phase between current $c$ and E. M. F. $e=u c$ consumed by the impedance $u$ is :

$$
\left.\begin{array}{l}
\cos \left(\begin{array}{ll}
c & e
\end{array}\right)=\frac{b}{u} \\
\sin \left(\begin{array}{ll}
c & e
\end{array}\right)=\frac{s}{u}
\end{array}\right\}
$$

Since the three E. M. F.'s acting in the closed circuit:

$$
\begin{aligned}
& e_{0}=\text { E. M. F. of generator, } \\
& e_{1}=\text { c. E. M. F. of synchronous motor, } \\
& e=u c=\text { E. M. F. consumed by impedance, }
\end{aligned}
$$

form a triangle, that is, $e_{1}$ and $e$ are components of $e_{0}$, it is (Fig. 1):

hence,

$$
e_{0}^{2}=e_{1}^{2}+e^{2}+2 e e_{1} \cos \left(e_{1}, e\right)
$$

$$
\cos \left(e_{1}, e\right)=\frac{e_{0}^{2}-e_{1}^{2}-e^{2}}{2 e_{1} e}=\frac{e_{0}^{2}-e_{1}^{2}-u^{2} c^{2}}{2 u c e_{1}},
$$

since, however, by diagram :

$$
\begin{aligned}
\cos \left(e_{1}, e\right)= & \cos \left(c, e-c, e_{1}\right) \\
& =\cos (c, e) \cos \left(c, e_{1}\right)+\sin (c, e) \cdot \sin \left(c, e_{1}\right)
\end{aligned}
$$

substitution of $(2),(3)$ and $(5)$ in (6) gives after some transposition :

$$
e_{0}^{2}-e_{1}^{2}-u^{2} c^{2}-2 r p=2 s \sqrt{c^{2} e_{1}^{2}-p^{2}},
$$

the Fundamental Equation of the Synchronous Motor, relating impressed E. M. F., $e_{0}$; c. E. M. F., $e_{1}$; current, $c$; power, $p$, and resistance, $r$; reactance, $s$; impedance, $u$.

This equation shows, that at given impressed E. M. F. $e_{0}$ and given impedance $u=\sqrt{r^{2}+s^{2}}$, three variables are left, $e_{1}, c, p$ of which two are independent. Hence, at given $e_{\mathrm{o}}$ and $u$, the current $c$ is not determined by the load $p$ only, but also by the excitation, and thus the same current $c$ can represent widely different loads $p$, according to the excitation; and with the same 
load, the current $c$ can be varied in a wide range, by varying the field excitation $e_{1}$.

The meaning of equation (7) is made more perspicuous by some transformations, which separate $e_{1}$ and $c$, as function of $p$. and of an angular parameter $\varphi$.

Substituting in (7) the new coordinates:

$$
\left.\begin{array}{l}
x=\frac{e_{1}^{2}+u^{2} c^{2}}{\sqrt{2}} \\
y=\frac{e_{1}^{2}-u^{2} e^{2}}{\sqrt{2}}
\end{array}\right\} \text { or, }\left\{\begin{array}{c}
e_{1}^{2}=\frac{x+y}{\sqrt{2}} \\
u^{2} e^{2}=\frac{x-y}{\sqrt{2}}
\end{array}\right\}
$$

we get

$$
e_{0}^{2}-x \sqrt{2}-2 \operatorname{rp}=2 \frac{s}{u} \sqrt{\frac{\bar{x}^{2}-y^{2}}{2}-u^{2} p^{2}}
$$

substituting again

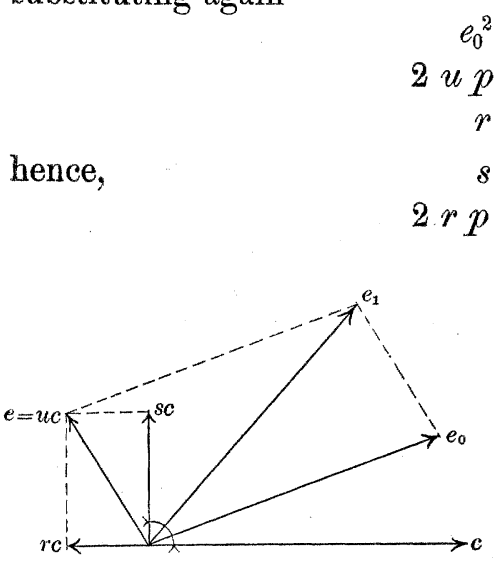

Fig. 1.

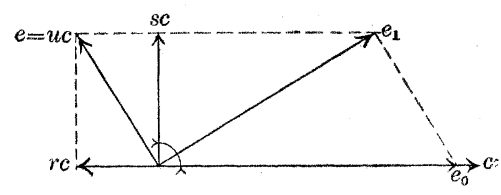

Fig. 2.

we get

$$
a-x \sqrt{2}-\varepsilon b=\sqrt{\left(1-\varepsilon^{2}\right)\left(2 x^{2}-2 y^{2}-b^{2}\right)}
$$

and, squared

$$
\varepsilon^{2} x^{2}+\left(1-\varepsilon^{2}\right) y^{2}-x \sqrt{2}(a-\varepsilon b)+\frac{b^{2}\left(1-\varepsilon^{2}\right)}{2}+\frac{(a-\varepsilon b)^{2}}{2}=0,
$$

substituting

gives, after some transposition,

$$
\left.\begin{array}{rl}
\varepsilon x-\frac{(a-\varepsilon b) \sqrt{2}}{2 \varepsilon} & =v, \\
y \sqrt{1-\varepsilon^{2}} & =w,
\end{array}\right\}
$$

$$
v^{2}+w^{2}=\frac{\left(1-\varepsilon^{2}\right)}{2 \varepsilon^{2}} a(a-2 \varepsilon b),
$$


hence, if

$$
R=\sqrt{\frac{\left(1-\varepsilon^{2}\right)(a-2 \varepsilon b) a}{2 \varepsilon^{2}}},
$$

it is

$$
v^{2}+w^{2}=R^{2}
$$

the equation of a circle with radius $R$.

Substituting now backwards, we get, with some transpositions: $\left\{r^{2}\left(e_{1}^{2}+u^{2} c^{2}\right)-u^{2}\left(e_{0}^{2}-2 r p\right)\right\}^{2}$

$$
+\left\{r s\left(e_{1}^{2}-u^{2} c^{2}\right)\right\}^{2}=s^{2} u^{2} e_{0}^{2}\left(e_{0}^{2}-4 r p\right) \text {. }
$$

the Fundamental Equation of the Synchronous Motor in a modified form.

The separation of $e_{1}$ and $c$ can be effected by the introduction of a parameter $\varphi$ by the equations :

$$
\begin{aligned}
& r^{2}\left(e_{1}^{2}-u^{2} c^{2}\right)-u^{2}\left(e_{0}^{2}-2 r p\right)=s u e_{0} \sqrt{e_{0}^{2}-4 r p} \cos \varphi \text { ) } \\
& \left.r s\left(e_{1}^{2}-u^{2} c^{2}\right)=s u e_{0} \sqrt{e_{0}^{2}-4 r p} \sin \varphi\right\}
\end{aligned}
$$

These equations (18), transposed, give

$$
\begin{aligned}
e_{1} & =\sqrt{\frac{1}{2}\left\{\frac{u^{2}}{r^{2}}\left(e_{0}^{2}-2 r p\right)+\frac{u e_{0}}{r}\left(\frac{s}{r} \cos \varphi+\sin \varphi\right) \sqrt{e_{0}^{2}-4 r p}\right\}} \\
& =\frac{e_{0}}{r} \sqrt{\frac{1}{2}\left\{\left(1-\frac{2 r p}{e_{0}^{2}}\right)+\left(\frac{s}{u} \cos \varphi+\frac{r}{u} \sin \varphi\right) \sqrt{\left.1-\frac{4 r p}{e_{0}^{2}}\right\}}\right.} \\
c & =\sqrt{\frac{1}{2}\left\{\frac{1}{r^{2}}\left(e_{0}^{2}-2 r p\right)+\frac{e_{0}}{r u}\left(\frac{s}{r} \cos \varphi-\sin \varphi\right) \sqrt{e_{0}^{2}-4 r p}\right\}} \\
& =\frac{e_{0}}{r} \sqrt{\frac{1}{2}\left\{\left(1-\frac{2 r p}{e_{0}^{2}}\right)+\left(\frac{s}{u} \cos \varphi-\frac{r}{u} \sin \varphi\right) \sqrt{1-\frac{4 r p}{e_{0}^{2}}}\right\}} .
\end{aligned}
$$

The Parameter Equations of the Synchronous Motor:-

The parameter $\varphi$ has no direct physical meaning, apparently. These equations (19) and (20), by giving the values of $e_{1}$ and $c$ as functions of $p$ and the parameter $\varphi$ enable us to construct the Power Characteristics of the Synchronous Motor, as the curves relating $e_{1}$ and $c$, for a given power $p$, by attributing to $\varphi$ all different values.

Since the variables $v$ and $w$ in the equation of the circle (16) are quadratic functions of $e_{1}$ and $c$, the Power Characteristics of the Synchronous Motor are Quartic Curves.

They represent the action of the synchronous motor under all conditions of load and excitation, as an element of power transmission even including the line, etc.

Before discussing further these Power Characteristics, some special conditions may be considered. 


\section{A. Maximum Output.}

Since the expression of $e_{1}$ and $c$ [equations (19) and (20)] contain the square root $\sqrt{e_{0}^{2}-4 r p}$, it is obvious that the maximum value of $p$ corresponds to the moment where this square root disappears by passing from real to imaginary, that is,

$$
\begin{gathered}
e_{0}^{2}-4 r p=0, \\
\text { or, } \quad p=\frac{e_{0}^{2}}{4 r}
\end{gathered}
$$

This is the same value, which represents the maximum power transmissible by E. M.F. $e_{0}$ over a non-inductive line of resistance $r$, or more generally, the maximum power which can be transmitted over a line of impedance $u=\sqrt{r^{2}+s^{2}}$ into any circuit, shunted by a condenser of suitable capacity.

Substituting (21) in (19) and (20), we get,

$$
\left.\begin{array}{l}
e_{1}=\frac{u}{2 r} e_{0} \\
c=\frac{e_{0}}{2 r}
\end{array}\right\}
$$

and the displacement of phase in the synchronous motor,

$$
\cos \left(e_{1}, c\right)=\frac{p}{c e_{1}}=\frac{r}{u}
$$

hence,

$$
\tan \left(e_{1}, c\right)=-\frac{s}{r}
$$

That is, the angle of internal displacement in the synchronous motor is equal but opposite to the angle of displacement of line impedance,

and consequently,

$$
\begin{aligned}
\left(e_{1}, c\right) & =-(e, c) \\
& =-(u, r)
\end{aligned}
$$

$$
\left(e_{0}, c\right)=0
$$

that is, the current $c$ is in phase with the impressed E. M. E. $e_{0}$. If $u<2 r, \quad e_{1}<e_{0}$ that is, motor E. M. F. $<$ generator Е. м. F. If $u=2 r, \quad e_{1}=e_{0}$ that is, motor E. M. F. $=$ generator Е. M. F. If $u>2 r, \quad e_{1}>e_{0}$ that is, motor Е. м. F. $>$ generator Е. м. Е. In either case, the current in the synchronous motor is leading.

B. Running Light, $\mathrm{P}=0$.

When running light, or for $p=0$, we get, by substituting in (19) and (20), 


$$
\begin{aligned}
& e_{1}=\frac{e_{0} u}{r} \sqrt{\frac{1}{2}\left\{1+\frac{s}{u} \cos \varphi+\frac{r}{u} \sin \varphi\right\}} \\
& c=\frac{e_{0}}{r} \sqrt{\frac{1}{2}\left\{1+\frac{s}{u} \cos \varphi-\frac{r}{u} \sin \varphi\right\}}
\end{aligned}
$$

Obviously this condition can never be fulfilled absolutely, since $p$ must at least equal the power consumed by friction, etc., and thus the true no load curve merely approaches the curve $p=0$, being however rounded off, where curve (26) gives sharp corners.

Substituting $p=0$ into equation (7) gives, after squaring: and transposing,

$e_{1}^{2}+e_{0}^{2}+u^{4} c^{4}-2 e_{1}^{2} e_{0}^{2}-2 u^{2} c^{2} e_{0}^{2}+2 r^{2} c^{2} e_{1}^{2}-2 s^{2} c^{2} e_{1}^{2}=0$.

This quartic equation can be resolved into the product of two quadratic equations,

$$
\left.\begin{array}{ll}
e_{1}^{2}+u^{2} c^{2}-e_{0}^{2}+2 s c e_{1}=0 & \text { generator. } \\
e_{1}^{2}+u^{2} c^{2}-e_{0}^{2}-2 s c e_{1}=0 & \text { motor. }
\end{array}\right\}
$$

which are the equations of two ellipses, the one the image of the other, both inclined with their axes.

The minimum value of c. Æ. M. F. $e_{1}$ is, $e_{1}=0$ at $c=\frac{e_{0}}{u}$

The minimum value of current $c$ is, $c=0$ at $e_{1}=e_{0}$

The maximum value of E. M. F. $e_{1}$ is given by equation (28),

$$
f=e_{1}^{2}+u^{2} c^{2}-e_{0}^{2} \pm 2 s c e_{1}=0
$$

by the condition,

hence,

$$
\frac{d e_{1}}{d c}=-\frac{\frac{d f}{d c}}{\frac{d f}{d e_{1}}}=0 \text { as, } u^{2} c \pm s e_{1}=0
$$

$$
c=e_{0} \frac{s}{r u}, e_{1}=\mp e_{0} \frac{u}{r} .
$$

The maximum value of current $c$ is given by equation (28) by

$$
\begin{aligned}
& \frac{d c}{d e_{1}}=0, \text { as } \\
& c=\frac{e_{0}}{r} \quad e_{1}=\mp e_{0} \frac{s}{r} .
\end{aligned}
$$

If as abscissæ $e_{1}$, and as ordinates $u c$ are chosen, the axes of these ellipses pass through the points of maximum power given by equation (22). 
It is obvious thus, that in the curves of synchronous motors running light, published by Mordey and others, the two sides of the V-shaped curves are not straight lines, as usually assumed, but arcs of ellipses, the one of concave, the other of convex curvature.

These two ellipses are shown in Fig. 3, and divide the whole space into six parts, the two parts $A$ and $A^{1}$, whose areas contain the quartic curves (19) (20) of synchronous motor, the two parts. $\mathbf{B}$ and $\mathbf{B}^{1}$, whose areas contain the quartic curves of generator,

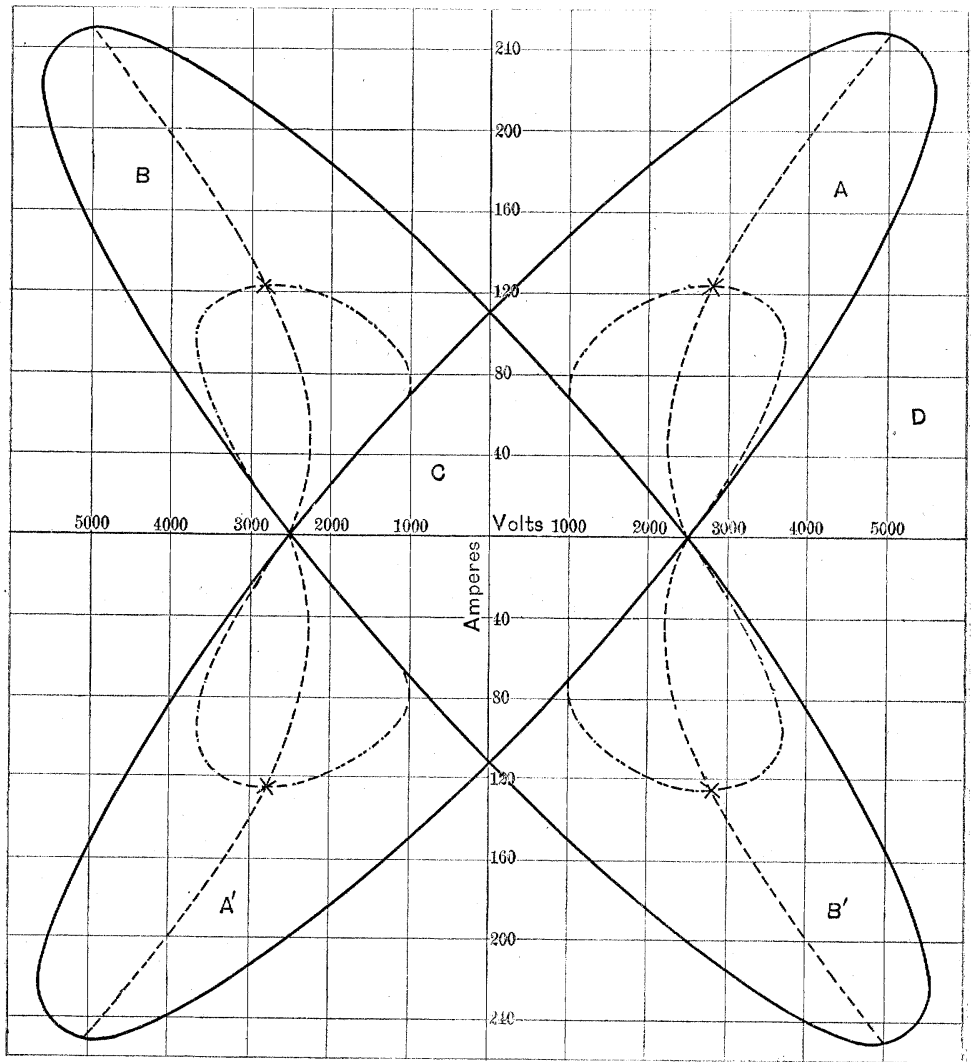

Fig. 3.

and the interior space $\mathrm{c}$ and exterior space $\mathrm{D}$, whose points do not represent any actual condition of the alternator circuit, but make the dependence $e_{1}, c$ imaginary.

$\mathbf{A}$ and $\mathbf{A}^{1}$, and the same $\mathbf{B}$ and $\mathbf{B}^{1}$, are identical conditions of the alternator circuit, differing merely by a simultaneous reversal of current and E. M. F., that is, differing by the time of a half period. 
Each of the spaces $\mathrm{A}$ and $\mathbf{B}$ contains one point of equation (22), representing the condition of maximum output of generator, viz., synchronous motor.

\section{Minimum Current at Given Power.}

The condition of minimum current, $c$, at given power, $p$, is determined by the absence of a phase displacement at the impressed w. M. F. $e_{0}$,

$$
\left(e_{0}, c\right)=0 \text { 。 }
$$

This gives from diagram Fig. 2,

$$
e_{1}^{2}=e_{0}^{2}+c^{2} u^{2}-2 c e_{0} \frac{r}{u}
$$

or, transposed,

$$
e_{1}=\sqrt{\left(e_{0}-c r\right)^{2}+c^{2} s^{2}}
$$

This quadratic curve passes through the point of zero current and zero power,

$$
c=0, \quad e_{1}=e_{0}
$$

through the point of maximum power (22),

$$
c=\frac{e_{0}}{2 r}, \quad e_{1}=\frac{e_{0} u}{2 r}
$$

and through the point of maximum current and zero power,

$$
c=\frac{e_{0}}{r}, \quad e_{1}=\frac{e_{0} s}{r}
$$

and divides each of the quartic curves or power characteristics into two sections, one with leading, the other with lagging current, which sections are separated by the two points of curve (34), the one corresponding to minimum, the other to maximum current.

It is interesting to note, that at the latter point the current can be many times larger than the current which would pass through the motor while at rest, which latter current is,

$$
c=\frac{e_{0}}{u}
$$

while at no load, the current can reach the maximum value,

$$
c=\frac{e_{0}}{r}
$$

the same value as would exist in a non-inductive cireuit of the same resistance. 
The minimum value at c. E. M. F. $e_{1}$, at which coincidence of phase, $\left(e_{0}, c\right)=0$, can still be reached, is determined from equation (34) by,

$$
\frac{d e_{1}}{d e}=0
$$

as,

$$
c=e_{0} \frac{r}{u^{2}} \quad e_{1}=e_{0} \frac{s}{u}
$$

The curve of no displacement, or of minimum current, is shown in Figs. 3 and 4 in dotted lines. ${ }^{1}$

\section{Maximum Displacement of Phase.}

$$
\left(e_{0}, c\right)=\text { maximum. }
$$

At a given power $p$ the input is,

hence,

$$
p_{0}=p+c^{2} r=e_{0} c \cos \left(e_{0}, c\right)
$$

$$
\cos \left(e_{0}, c\right)=\frac{p+c^{2} r}{e_{0} c}
$$

At a given power $p$, this value, as function of the current $c$, is a maximum when

this gives,

$$
\frac{d}{d c}\left(\frac{p+e^{2} r}{e_{0} c}\right)=0
$$

or,

$$
p=c^{2} r
$$

$$
c=\sqrt{\underline{p}}
$$

That is, the displacement of phase, lead or lag, is a maximum, when the power of the motor equals the power consumed by the resistance, that is, at the electrical efficiency of 50 per cent.

Substituting (40) in equation (7) gives, after squaring and transposing, the Quartic Equation of Maximum Displacement,

$$
\begin{aligned}
\left(e_{0}^{2}-e_{1}^{2}\right)^{2}+c^{4} u^{2}\left(u^{2}+8 r^{2}\right)+ & 2 c^{2} e_{1}^{2}\left(5 r^{2}-u^{2}\right) \\
& -2 e^{2} e_{0}^{2}\left(u^{2}+3 r^{2}\right)=0
\end{aligned}
$$

1. It is interesting to note, that the equation (34) is similar to the value, $e_{1}=$ $\sqrt{\left(e_{0}-c r\right)^{2}-c^{2} s^{2}}$, which represents the output transmitted over an inductive line of inpedance $u=\sqrt{r^{2}+s^{2}}$ into a non-inductive circuit.

Equation (34) is identical with the equation giving the maximum voltage $e_{1}$, at current $c$, which can be produced by shunting the receiving circuit with a condenser, that is, the condition of "complete resonance" of the line $u=$ $\sqrt{r^{2}+s^{2}}$ with current $c$. Hence, referring to equation (35), $e_{1}=e_{0} \frac{s}{r}$ is the maximum resonance voltage of the line, reached when closed by a condenser of reactance, $-s$. 
The curve of maximum displacement is shown in dash-dotted lines in Fig. 3 and 4 . It passes through the point of zero current -as singular or nodal point-and through the point of maximum power, where the maximum displacement is zero, and it intersects the curve of zero displacement.

E. Constant Counter E. M. F.

At constant 0. E. M. F., $e_{1}=$ constant,

If,

$$
e_{1}<e_{0} \sqrt{1-\frac{r^{2} x^{2}}{u^{4}}}
$$

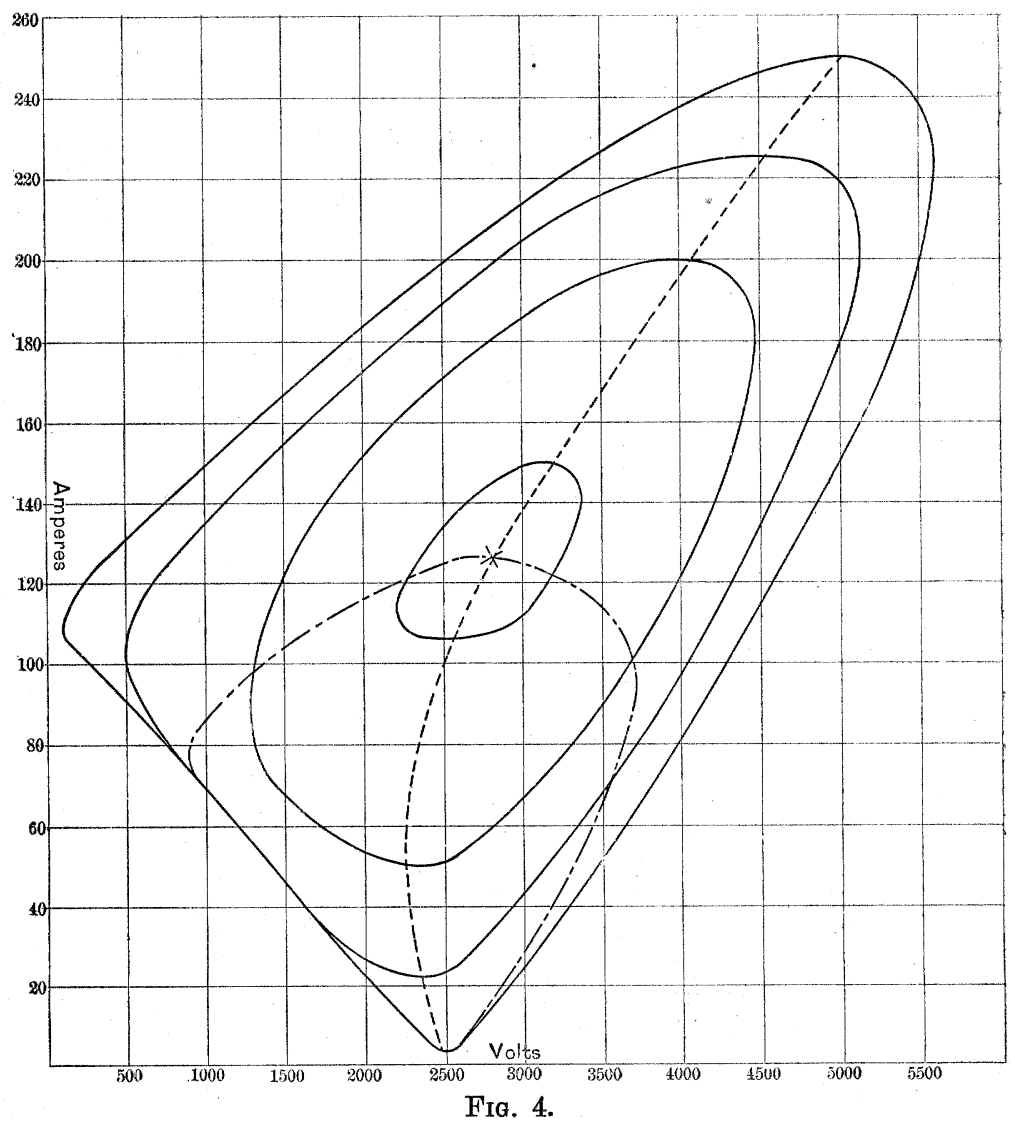

the current at no load is not a minimum, and is lagging. With increasing load, the lag decreases, reaches a minimum, and then increases again, until the motor falls out of step, without ever 
coming into coincidence of phase.

If,

$$
e_{0} \sqrt{1-\frac{r^{2} s^{2}}{u^{2}}}<e_{1}<e_{0}
$$

the current is lagging at no load; with increasing load the lag decreases, the current comes into coincidence of phase with $e_{0}$, then becomes leading, reaches a maximum lead; then the lead decreases again, the current comes again into coincidence of phase, and becomes lagging, until the motor falls out of step.

If $e_{0}<e_{1}$, the current is leading at no load, and the lead first increases, reaches a maximum, then decreases, and whether the current ever comes into coincidence of phase, and then becomes lagging, or whether the motor falls out of step while the current is still leading, depends, whether the o. E. M. F. at the point of maximum output is $>e_{0}$ or $<e_{0}$.

\section{F. Numerical Instance.}

Figs. 3 and 4 shows the characteristics of a 100 K.w. motor, supplied from a 2500-volt generator over a distance of 5 miles, the line consisting of two wires, No. 2 B. and S. G., 18 inches apart.

In this case we have,

$$
\begin{aligned}
& e_{0}=2500 \text { volts constant at generator terminals. } \\
& r=10 \text { olhms, including line and motor. } \\
& s=20 \text { ohms, including line and motor. }
\end{aligned}
$$

hence, $u=22.36$ ohms.

Substituting these values, we get,

$$
2,500^{2}-e_{1}^{2}-500 c^{2}-20 p=40 \sqrt{c^{2} e_{1}^{2}-p^{2}}
$$

$$
\begin{aligned}
\left\{e_{1}^{2}+500 o^{2}-31.25 \times 10^{6}+100 p\right\}^{2} & \\
+ & \left\{2 e_{1}^{2}-1,000 c^{2}\right\}^{2}=7.8125 \times 10^{15}-5+10^{9} p .
\end{aligned}
$$

$e_{1}=5590$ $0=559$

$\sqrt{\frac{1}{2}\left\{\left(1-3.2 \times 10^{-6} p\right)+(.894 \cos \varphi+.447 \sin \varphi) \sqrt{\left.1-6.4 \times 10^{-6} p\right\}} .\right.}$

$\sqrt{\frac{1}{2}\left\{\left(1-32 \times 10^{-6} p\right)+\left(894 \cos \varphi-.447 \sin \varphi \sqrt{\left.1-6.4 \times 10^{-6} p\right\}} .\right.\right.}$ Maximum output,

at,

$$
\left.\begin{array}{l}
p=156.25 \text { k.w. } \\
e_{1}=2795 \text { volts } \\
c=125 \text { amperes }
\end{array}\right\}
$$

Running light,

$$
\left.\begin{array}{c}
e_{1}^{2}+500 c^{2}-6.25 \times 10^{4} \mp 40 c e_{1}=0 \\
\dot{B}_{1}=20 c \pm \sqrt{6.25 \times 10^{4}}-100 o^{2}
\end{array}\right\}
$$


At the minimum value of $\mathrm{r}$. Е. м. F. $e_{1}=0$, is. $c=112$

At the minimum value of current, $c=0$, is, $e_{1}=2500(30)$ At the maximum value of c.E.M.F. $e_{1}=5590$ is, $c=223.5(31)$, At the maximum value of current, $c=250$, is, $e_{1}=5000(32)$ Curve of zero displacement of phase,

$$
\begin{aligned}
e_{1} & =10 \sqrt{(250-c)^{2}+4 c^{2}} \\
& =10 \sqrt{6.25 \times 10^{4}-500 c+5 c^{2}}
\end{aligned}
$$

Minimum c. E. M. F. point of this curve,

$$
c=50 \quad e_{1}=2240
$$

Curve of maximum displacement of phase,

$$
\begin{gathered}
p=10 c^{2} \\
\left(6.25 \times 10^{6}-e_{1}^{2}\right)^{2}+.65 \times 10^{6} c^{4}-10^{10} c^{2}=0
\end{gathered}
$$

Fig. 3 gives the two ellipses of zero power, in drawn lines, with the curves of zero displacement in dotted, the curves of maximum displacement in dash-dotted lines, and the points of maximum power as crosses.

Fig. 4 gives the Motor Power Characteristics, for,

$$
\begin{array}{ll}
p=10 & \text { к. } \mathrm{w} . \\
p=50 & \text { к. } \mathrm{w} . \\
p=100 & \text { к. } \mathrm{w} . \\
p=150 & \text { к. } \mathrm{w} . \\
p=156.25 & \text { к. } \mathrm{w} .
\end{array}
$$

together with the curves of zero displacement, and of maximum displacement.

\section{G. Discussion of Results.}

The characteristic curves of the synchronous motor, as shown in Fig. 4, have been observed by me frequently, with their essential features, the V-shaped curve of no load, with the point rounded off and the two legs slightly curved, the one concave, the other convex; the increased rounding off and contraction of the curves with increasing load; and the gradual shifting of the point of minimum current with increasing load, first towards lower, then towards higher values of c. F. M. F. $e_{1}$.

The upper parts of the curves however I have never been able to observe experimentally, and consider it as probable, that they correspond to a condition of synchronous motor running, which is unstable. The experimental observations usually extend about over that part of the curves of Fig. 4, which is reproduced in Fig. 5 , and in trying to extend the curves further to either side, the motor is thrown out of synchronism. 
It must be understood, however, that these power characteristics of the synchronous motor in Fig. 4 can be considered as approximations only, since a number of assumptions are made, which are not, or only partly fulfilled in practice. The foremost of these are:

1. It is assumed that $e_{1}$ can be varied unrestrictedly; while in reality the possible increase of $e_{1}$ is limited by magnetic saturation. Thus in Fig. 4 , at an impressed E. M, F. $e_{0}=2,5: 0$ volts, $e_{1}$ rises up to 5,590 volts, which may or may not be beyond that which can be produced by the motor, but certainly is beyond that which can be constantly given by the motor.

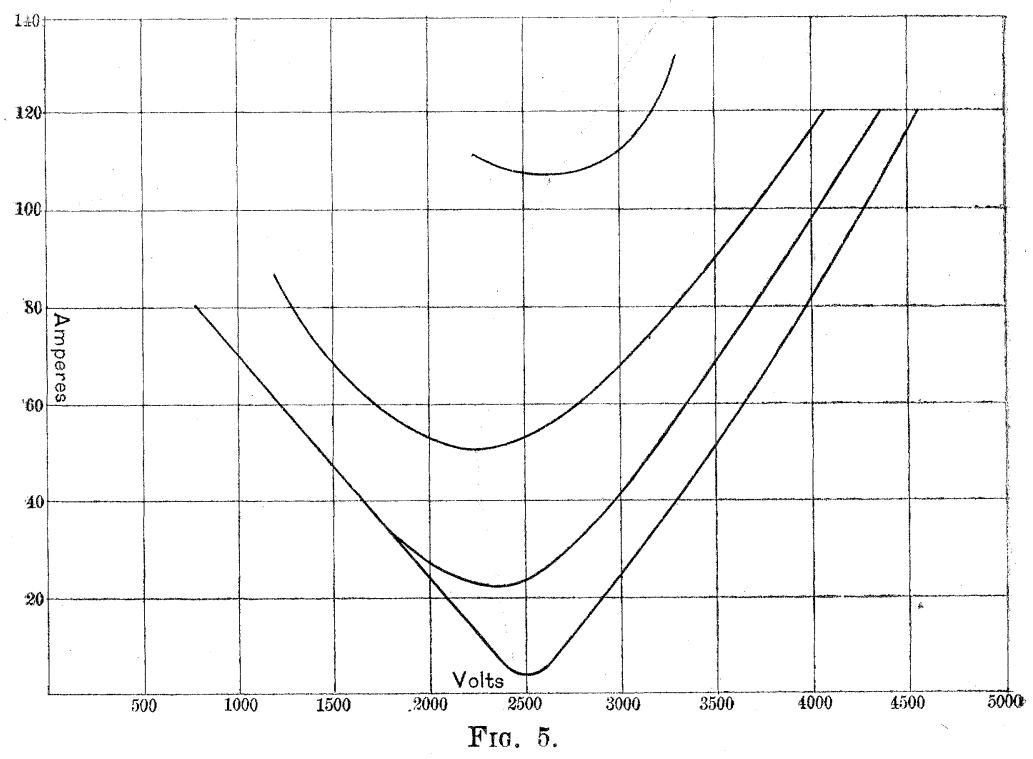

2. The reactance $s$ is assumed as constant. While the reactance of the line is practically constant, that of the motor is not, but varies more or less with the saturation, decreasing for higher values. This decrease of $s$ increases the current $c$ corresponding to higher values of $e_{1}$, and thereby bends the curves upwards at a lower value of $e_{1}$ than represented in Fig. 4.

It must be understood that the motor reactance is not a simple quantity, but represents the combined effect of self-induction, that is, the E. M. F. induced in the armature conductor by the current flowing therein, and armature reaction, that is, the variation of the o. .. м. F. of the motor by the change of the resultant 
field, due to the superposition of the M. M. F. of the armature current upon the field excitation.

3. These curves in Fig. 4 represent the conditions of constant electric power of the motor, thus including the mechanical and the magnetic friction (core loss). While the mechanical friction can be considered as approximately constant, the magnetic friction is not, but increases with the magnetic induction, that is with $e_{1}$, and the same holds for the power consumed for field excitation.

Hence the useful mechanical output of the motor will on the same curve $p=$ const. he larger at points of lower c. E. м. F. $e_{1}$, than at points of higher $e_{1}$, and if the curves are plotted for constant useful mechanical output, the whole system of curves will be shifted somewhat towards lower values of $e_{1}$, hence the points of maximum output of the motor correspond to a lower $\mathrm{E}$. M. F. also.

It is obvious that the true mechanical power characteristics of the synchronous motor can be determined only in the case of the particular conditions of the installation under consideration. 


\section{Discussion in New York.}

Mr. Kennelly :- This subject is so very interesting and it is capable of such very beautiful expression, that if $\mathrm{Mr}$. Steinmetz will pardon me for making room on the blackboard, I think it might interest some of those who have followed Mr. Steinmetz's able exposition of it to see the same results and the same treatment presented under a different point of view.

III The fundamental relations of the continuous current motor are two, namely an E. M. F. relation and an activity relation. If

$E$ be the impressed E. M. F. at armature terminals (volts)

$e$ " counter E. M. F. in the armature (volts)

$R$ " resistance of the armature (ohms)

$I$ " current through the armature (amperes)

$P \quad$ " activity absorbed electro-dynamically by the armature (watts).

Then the drop in the armature resistance must be $(E-e)$ volts, and this divided by the resistance of the armature must give the current in the motor by Ohm's law, so that

$$
\frac{E-e}{R}=I \text {. }
$$

The product of the current and the c. ж. M. F. of the motor must be the activity absorbed by the armature and expended in mechanical output and in overcoming frictions of every kind, so that

$$
\text { e } I=P \text {. }
$$

The c. E. M. F. e depends upon the speed and excitation. For a given excitation the speed of the armature automatically adjusts itself until the right amount $I$ of current passes through, to enable $e$ l just to absorb the power needed for output plus frictions.

In the synchronous alternating current motor we have the same equations, employing the same terms, but with their meaning slightly extended to suit the changed condition. The speed of the armature cannot now change, assuming the generator to run at a constant speed, and the c. E. м. F. of the armature depends entirely on the excitation. If the o. E. M. F. Was restricted to being in phase with the impressed E. M. F., it is clear that with equations (1) and (2) could only be satisfied by giving just the right excitation, and that the relations would be unstable, for any change in the load would upset the relation until a new and correct tield excitation had been given to make $e$ properly limit the entering current. But if, as is found to be the case, the c.E.M. F. $e$ can differ in phase from $E$, a new means of automatic adjustment is provided. The resistance $R$ of the armature becomes, in the case of an alternating current circuit, an impedance $J$ (ohms). Also, when the current and the c. E. M. F. are out of step, the activity $P$ is not their simple numerical product, but 
their co-directed product, i.e., the product of one and the resolved part of the other in its direction, viz.:

$$
\text { e } I \cos \alpha=P
$$

$\alpha$ being the angle of phase difference. The fundamental equations of a synchronous motor are, therefore,

$$
\begin{gathered}
\frac{E-\varepsilon}{J}=I \\
e I \cos \alpha=P .
\end{gathered}
$$

In equation (3) $E \varepsilon J$ and $I$ are all vector quantities, while in equation (4) all the quantities are numerics only, $e$ and $I$ being the number of volts and amperes in $\varepsilon$ and $I$ respectively, without regard to direction. The consequences of these relations are most clearly manifested graphically.
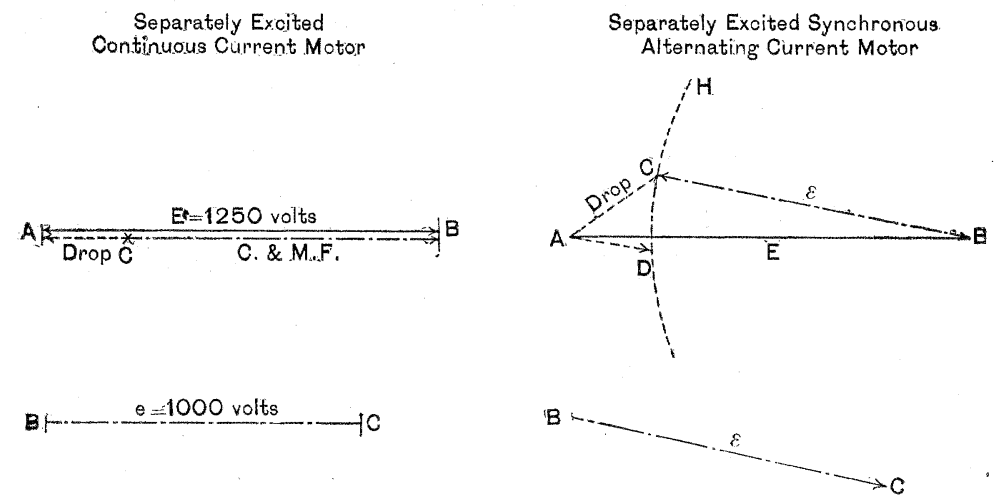

Fig. 1.

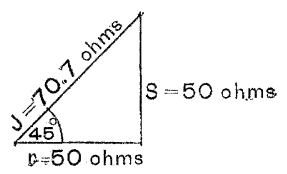

Fig. 2.

If we take a 1,250 volt continuous current motor of $50 \mathrm{ohms}$ armature resistance, and let it run under a load such that its. mechanical output is $4 \mathrm{kw}$., then the losses of activity in friction, hysteresis and eddies (by measurement or computation) might be $5 \mathrm{kw}$., making the power absorption $P=5 \mathrm{k}$. w. and the current in the armature 5 amperes. The drop in the armature will be 250 volts, and the o. E. M. F. 1,000 volts.

If the line $\mathrm{A} \mathrm{B}$ in Fig. 1 represents 1,250 volts to scale, and the line $\mathrm{B} \mathrm{c}$ beneath 1,000 volts, then $E-e$ will be $\mathrm{A}$ c, or 250 volts, and this divided by the resistance of the armature $50 \mathrm{ohms}$. gives 5 amperes the current in the motor.

Taking now the corresponding case of an alternating synchronous motor, A B (Fig. 2) represents the E. M. F. $\varepsilon$ of 1,250 volts at. 
terminals. The C. E. M. F. $\varepsilon$ will not usually be in phase with this, but may have an inclined direction, such as B c, beneath, of 1,000 volts magnitude, this C. $\mathrm{k}$. M. F. will, neglecting the secondary effects of armature reaction, remain constant at all loads when the excitation of the field magnets is constant. $E-\varepsilon$ will therefore be A в - в c or A c, Fig. 2, shown as 354 volts, and this will be the drop in the armature, instead of 250 volts in the continuous current case. To find the current, we divide a c by the impedance of the armature. If the armature resistance $r=50 \mathrm{ohms}$, and its reactance $s=$ say $50 \mathrm{ohms}$, the impedance will be $J=70.7 \angle 45^{\circ} \mathrm{ohms}$, as shown in Fig. 2. If we divide this into A $\mathrm{C}$, we not only obtain the numerical quotient $\frac{353.5}{70.7}=5$ amperes, but we lay off this quotient along A inclined at the impedance angle $45^{\circ}$ from $\mathrm{A} \mathrm{c}$, in accordance with the rules of vector division. In this case $\mathrm{A}$ в happens to be in phase with or parallel to B $\mathrm{C}$ the C. E. M. F., so that their co-directed product is their simple numerical product $5 \times 1000=5 \mathrm{k}$.w. the activity mechanically absorbed by the armature. Usually, however, they will not be parallel, but will include some angle $\alpha$ less than $90^{\circ}$. The phase angle between $\varepsilon$ and $E$ will therefore be such that the vector drop A $\mathrm{c}$, which must connect $\mathrm{A}$ with some point on the circle н о к of radius $\varepsilon$, when divided by the vector impedance $J$, gives a current whose co-directed product with $\varepsilon$ gives the activity which the motor requires at its imposed torque.

The separately excited continuous current motor adjusts its current by its speed and c. E. M. F. The separately excited synchronous motor cannot alter its speed or c. E. M. F., but adjusts its current by its phase of C. E. M. F. By varying the magnet excitation and, therefore, the c. E. м. F. of the armature, the phase angle of the current for a constant load can be widely altered. It can be made to lag behind, to run before, or to keep step with the impressed E. M. F.

Dr. Pupin:- I would like to add one or two remarks to what I have already said, merely as a comment to Mr. Steinmetz's remarks. The differences between Mr. Steinmetz's method and that of Lieutenant Reber are essential if I understand Mr. Steinmetz correctly. The practical results to which they lead will, of course, be the same. They can be stated as follows:- that Mr. Steinmetz defines his quantities by calling them certain names. He calls a thing "impedance" and defines it by saying that it is the product of an electromotive force multiplied by a current. The reactance is then defined by the relation $u^{2}=r^{2}+s^{2}$ where $r$ is the resistance, $s$ the reactance and $u$ the impedance. The electromotive forces, and the currents are given by the amplitudes. The back electromotive force is the E. M. F. of the motor. These definitions are non-committal and therefore Mr. Steinmetz's results will be true even if hysteresis, Foucault current, and magnetic leakage are present. But if they are to hold true in the pres- 
ence of these, then the quantities which they define are only experimentally measurable quantities, and cannot be calculated by a general formula; as we can calculate, for instance, the coefficient of salf-induction of a coil surrounding a closed magnetic circuit in terms of the number of turns, and the reluctance of the magnetic circuit, when the modifying effects due to the Foncault currents and magnetic leakage are neglected.

Of course to a practical engineer who wishes to consult the results only, Mr. Steinmetz's results are just as valuable as any other. But to a designer or to an inventor, or to an investigator who wishes to go beyond the actual results, and inquire into the causes of armature reactions, magnetic leakages, variations of permeability, etc., the theory which speaks in terms of those quantities as defined in Lieut. Reber's paper seems to me to be much preferable. Besides the fundamental theory of a new apparatus like the motor should be written in terms of the fundamental quantities, that is resistance, co-efficient of self-induction, and capacity, as ideally defined. It is a theory of an important practice, and it is intended to be the foundation on which the members of this InstrTute are going to build, and in the course of this building up it will be time enough to take care of hysteresis, Foucault currents and magnetic leakage. If you look at the question from the fundamental standpoint, you must take an ideal view of it, and therefore you must treat the fundamental quantities like resistance, self-induction, and capacity in an ideal sense. The theory so constructed, as I said before, will serve two purposes. It will express relations of the fundamental quantities in an ideal, and therefore perfectly definite sense; and secondly, it will give you a hint why in a given case practical results differ from the purely ideal relations. It will give you hints which may lead to discovery of new properties of the material of which the machine is constructed. It will assist you in investigating the properties of the various parts of electric and magnetic circuits-the properties which you did not take into consideration in the ideal theory, but the existence of which will in all probability bring about practical results differing from the ideal theory. I think that this is a very important thing to consider in theoretical discussions.

There is another reason why, in my opinion, Lieut. Reber could not adopt Mr. Steinmetz's method. If he had adopted this method, I do not see how he could have considered the quantity $K$ to which he attaches so much importance: namely, the relation between the reactance and the resistance; and how could he, for instance, arrive at the result that it is a very good thing to introduce a resistance or capacity in a motor if you want to vary its torque. So that I do not agree with Mr. Kennelly or with Mr. Steinmetz that because one theory seems to be formally simpler than another, that it is more desirable. One theory may be good for one man, the other theory is good for another man, and that 
theory which is to serve as the fundamental theory, say, of an induction motor, must take into consideration, not the mean value of experimentally determinable quantities, like impedance, counter-electromotive force, etc., but the fundamental quantities, the ideal resistance, the self-induction, and the capacity, and that too the values of these quantities for ideal conditions, for it is under these conditions only, that we can calculate beforehand the numerical values of these quantities.

Mr. Steinmetz:- I think Prof. Pupin misunderstood, due to the shortness of what I said. These equations of the induction motor, which I wrote here, giving the torque and the power, did not contain the impedance, but contained the resistance and the reactance. The quantity, $s$, is nothing else than $=2 \pi N L$. As long as the frequency is the same, the reactance remains the same, and in the impedance of the armature I used resistance and reactance separately, and in the latter introduced the slip so as not to get a variable quantity, but reduced to reactance at standstill.

Thus all the quantities used by me are reduced to the fundamental constants, resistance, inductance, capacity--which is absent in an induction motor-and coefficient of hysteresis. The armature resistance is contained explicitly in the equation, and it was from these very equations that $I$ investigated its effect. Therefore, the exceptions made by Dr. Pupin do not apply to my theory.

Dr. Pupin:-But you do not specify that you are neglecting. leakage and hysteresis; as long as you do not do that, so long will $s$ be not the reactance that can be expressed in number of turns, length, cross-section, and permeability of the magnetic circuit, etc., by the well-known rules, but will be a quantity that can be determined by actual experimental test only.

Mr. Strinmerz:- I do not think that is so. When I want to build a 100-horse power induction motor, if I should build it first and then determine by actual test its reactance, etc., I do not think that such a way of designing would do for a long time. All these quantities are determined by calculations beforehand, and when I said that I build the induction motor so as to give a starting torque 50 per cent. in excess of running torque, this is not done by running the motor, and experimentally determining what resistance gives the desired torque, but the resistance is calculated before the motor is built, and the test of the finished motor merely checks the calculation.

Dr. Pupin :- If you take these values, you ought to say in the beginning of the paper that you are neglecting hysteresis and leakage.

Mr. Steinmetz:-No. Hysteresis is not neglected.

Dr. PUPIN :-No man in the world can calculate the coefficient of self-induction of a varying magnetic circuit in which the permeability and the distribution of magnetic induction is continually varying. 
Mr. Steinmetz:- The permeance does not vary. The iron in an induction motor necessarily is worked at a low magnetic induction, where its permeability is so high, that the reluctance of the magnetic circuit is practically all the reluctance of the airgap. I can show you such motor magnetization curves, where I had the same induction motor running at various magnetic inductions, that is, at various impressed electromotive forces. The curve of current, that is, of м. M. F., as function of the ะ. M. F., that is the magnetic induction, is practicaliy a straight line, which extended, passes through the origin. That is, the magnetic reluctance is practically constant.

Dr. Pupin :- If that is so, and I think it is so, as you say, then hysteresis and the variation of the magnetic permeability and the variation of the magnetic leakage with the load does not amount to quite as much as you led me to infer in your discussion of $\mathrm{Mr}$. Reber's paper.

Mr. Sternmetz:-No. That is a different thing. The hysteresis may vary, and so may vary the magnetic stray field, and the useful flux. But the reactance has nothing to do with hysteresis. It is entirely independent. The magnetic leakage means the magnetic flux passing between armature and field, between primary and secondary circuits. If you take an ordinary induction motor and have no resistance in the armature, a highly efficient induction motor, then at standstill more than half the flux passes between the primary and secondary, and the product of the two $L$ 's is very greatly different from $M^{2}$. It is nearly four times as large.

Coming now to the theory of the synchronous motor, the quantities introduced in the equations are: the impressed E. M. F., the counter E. м F., and the impedance. The counter E. M. F. is the E. M. F. induced in the armature conductors by their rotation through the magnetic field, thus is derived from the magnetic characteristic of the machine, which can be determined experimentally or calculated as known. The impedance is composed of the resistance, $r$, and the reactance, $s$.

The only quantity which is variable and of complex nature is the reactance of the motor proper, which includes the self-induction and the armature reaction. Thus this quantity has been specially discussed by me in the last chapter.

I have tried to reduce all the quantities entering the equations to their elementary constants, which can be determined experimentally, and which can be calculated beforehand, because a theory of any machine is very nice and of high scientific interest, but to be useful, the first condition is that you can determine all the quantities which enter into the theory beforehand by calculation, the second condition is, that you can easily determine these quantities by experiment to check the correctness of your calculation, and the third condition is that you can easily recognize the limits of the practical application of your theory.

[Adjourned.] 